the method of presentation of the material in this volume are not on a graduate level. . .." Indeed, this could not possibly be so in a single volume which includes: A.c. vector theory; bridge and other measurements ; transient analysis ; network theory ; wave-filters; thermionic emission; electron dyna. mics; transmitter and receiver circuits; gasdischarge tubes; modulation theory; timing, rectification and control circuits. The treatment is essentially technological and, in many places, merely descriptive.

The recent flood of books on the more technological aspects of electrical communications makes one search any new publication for positive contributions to the art; in this respect, this book is saved by certain novel treatments. In particular, attention may be directed to the use of surface models in connexion with coupled circuits, and to the simple approach to the problem of transients and signal distortion.

Against these favourable points must be weighed the very condensed and sketchy treatment of certain topics. Thus, magnetrons are dealt with in one page, while klystron and other micro-wave tubes are omitted (in spite of the title). Transmission line theory has been included in a companion volume dealing also with wave-guides and antennæ, and this has necessitated treating wave-filters from the beginning as finite structures, which, though the more practical condition, results in an unwieldy presentation likely to be confusing to the beginner.

To two appendixes are relegated the elements of the relevant mathematics, electricity and magnetism : Appendix (a) opens with the definition of powers of a number and meaning of logarithms, and Appendix (b) with the inverse square law. If it be assumed that the reader needs to start at such elementary levels, it is unlikely that he will learn much from such intense cramming. It would have been preferable to have used this space for expanding the main text, giving references to good elementary text-books which might supply the foundations.

The urgency of the War required the rapid training of great numbers of people, with varying backgrounds, in the application of electronic techniques. But the value of such a course is doubtful now. Nevertheless, there must be many scientific people, possessing a suitable grounding in mathematics, electricity and magnetism, who may be interested in applying such techniques to their particular branch of science. For them, this volume may serve as a reference book and as a guide to the literature. There are a few, but select, references and a clear index; the diagrams are good, and the style is consistent in spite of the joint authorship.

E. C. Cherry

\section{METABOLISM OF DRUGS}

\section{Detoxication Mechanisms}

The Metabolism of Drugs and Allied Organic Compounds. By Dr. R. Tecwyn Williams. Pp. viii +288 . (London: Chapman and Hall, Ltd., 1947.) 25s. net.

HIS is the first monograph to be published in

English describing the chemical changes which drugs or other substances foreign to the body undergo whon administered to animals. It is undoubtedly a useful compendium of the work which has been carried out in this field. Dr. R. T. Williams excuses his title on the grounds that the subject has been treated under such a heading in text-books of biochemistry. The title is, however, too narrow for the subject. Many of the reactions considered are certainly not detoxication processes. Thus the conversion of the azo dye prontosil into sulphanilamide appears to be an activation, and the metabolism of some substances such las picric acid and trinitrotoluene leads to more toxic products. The functions of the biochemical changes are unknown in most cases. "Detoxication Mechanisms" is rather a teleological expression, and the reviewer would like to suggest that another title or even the present sub-title be used for future editions. Even if it has become customary to use this term, there is no need to continue using a term which Dr. Williams admits if "Taken in its literal sense . . . becomes in many cases a misnomer".

The metabolic processes which are described include oxidation and reduction and synthetic reactions such as the formation of thiocyanate from cyanide of acetyl and methyl derivatives of amines, and conjugation with glucuronic acid, sulphate, glycine, acetyl cysteine, ornithine and glutamine. The changes are considered particularly in reference to different groups of compounds, according to whether they are aliphatic or aromatic and the nature of their substituent groups.

The changes which most normal body constituents undergo, such as phosphorylation or oxidation, are often the same in all species, from bacteria to the higher mammals. The changes undergone by drugs and purines, however, show remarkable differences. Thus phenylacetic acid is conjugated with glycine by most mammals but with glutamine by men and chimpanzees and with ornithine by fowls. Aromatic amines are acetylated by most animals and birds, but not by dogs. The metabolism of $\beta$-naphthylamine shows interesting differences. When given to dogs, in which species it induces cancer of the bladder, it is excreted as 1-hydroxy, 2 -amino derivatives; but in rats, rabbits and monkeys, in which bladder cancer has never been induced with this compound, 6-hydroxy, 2-acetylamino-naphthalene is produced.

The biochemical changes which synthetic substances undergo in the body give an indication of capabilities which would otherwise pass unrecognized. Thus the acetylation of amines is most easily demonstrated with foreign substances such as the sulphonamides. The study of the metabolism of phenylsubstituted fatty acids gives indications of the metabolism of fatty acids in the body. In this case the phenyl group acts as a tracer. Dr. Williams considers that there is no satisfactory unitary theory for the processes considered. Substances foreign to the body are probably changed chemically because they resemble natural body constituents sufficiently closely for them to become substrates of existing enzymes. A fresh drug may stimulate the body to produce a fresh enzyme by adaptation, but in many cases animals metabolize foreign compounds as soon as they are administered.

Dr. Williams has produced a useful compendium of the facts of the subject which will be of value to many biologists with interests in pharmacology and chemotherapy. When more facts are known, it might be possible to generalize and introduce satisfactory hypotheses for the innumerable chemical processes which the animal body can carry out.

E. BoYLaND 